\title{
The current role of liver surgery in the treatment of colorectal liver metastases
}

\author{
Stefan Heinrich \\ General, Visceral and Transplantation Surgery, University Hospital of Mainz, Mainz, Germany \\ Correspondence to: Stefan Heinrich, MD. General, Visceral and Transplantation Surgery, University Hospital of Mainz, Langenbeckstrasse 1, 55131 \\ Mainz, Germany. Email: stefan.heinrich@unimedizin-mainz.de. \\ Comment on: Imai K, Benitez CC, Allard MA, et al. Impact of Surgical Treatment for Recurrence After 2-Stage Hepatectomy for Colorectal Liver \\ Metastases, on Patient Outcome. Ann Surg 2019;269:322-30.
}

Submitted Apr 30, 2019. Accepted for publication May 08, 2019.

doi: 10.21037/hbsn.2019.05.02

View this article at: http://dx.doi.org/10.21037/hbsn.2019.05.02

Since the establishment of a survival benefit for patients with colorectal cancer liver metastases (CRLM) undergoing liver resection, perioperative outcome has continuously improved, and surgery for CRLM is currently offered with a mortality below $1 \%$ in specialized centers (1). Following this development, liver surgery is considered the main curative treatment option for CRLM by most experts, and thus, the indications for surgery of CRLM are continuously extended. These extensions of surgical indications have been based on (I) increasing efficacy of systemic chemotherapy, (II) technical improvements of liver surgery and (III) the expanding knowledge of liver regeneration. Several modern surgical concepts such as repeat or staged resections and portal vein manipulations are based on the unique potential of the liver to regenerate its volume after tissue loss or damage.

Repeat liver resections for recurrent CRLM can be performed with a perioperative mortality and a longterm survival comparable to primary liver resections (2). In theory, the number of repeat resections is unlimited. However, previous resections of major hilar structures or hepatic veins limit repeat resections. Similarly, staged resections can be performed for extensive (bilateral) CRLM: after clearing one side of the liver from metastases by atypical resections, further resections can be added to resect remnant metastases after an adequate hypertrophy of the liver remnant (1). Since the occlusion of major branches of the portal vein triggers the same hypertrophy in the contralateral lobe as a resection of the corresponding liver volume, embolization (PVE) or ligation (PVL) of such portal venous branches are used to increase the future liver remnant (FLR) in combination with single or staged hepatectomies (1). The most pronounced and fastest volume increase is achieved by the Associating Liver Partition and Portal Vein Ligation for Staged Hepatectomy (ALPPS) concept, which achieves a volume increase of more than $150 \%$ within 7-10 days (3). While staged hepatectomies with PVE/PVL inherit a $25 \%$ drop-out risk due to disease progression before the second stage, all patients achieve complete resections by ALPPS, which in turns is associated with an earlier tumor recurrence (3).

In addition to these technical achievements, modern antibody-based chemotherapy regimen achieve significant response rates. By reducing the tumor volume, the FLR relatively increases, and single or staged hepatectomies can be offered with excellent oncological outcome. In this light, patients with a significant tumor response exhibit the best long-term survival after a curative liver resection, and those responding to chemotherapy even benefit from R1-resections. Moreover, recent analyses suggest that R1situations due to direct contact to vascular structures may have a survival comparable to R0 resections (4). In general, parenchyma-preserving resections with minimal resection margins are the basis for curative surgery of CRLM today, while a margin of more than $1 \mathrm{~cm}$ and anatomical resections had been standard in the past.

Furthermore, laparoscopic surgery has recently proven to be superior over open liver surgery in the setting of 
parenchyma-preserving liver surgery in terms of a lower complication rates, shorter hospital stay and an improved quality of life (5). Moreover, a recent match-pair analysis revealed a significantly earlier start of chemotherapy after laparoscopic over open liver resections for CRLM (6). These beneficial effects of laparoscopic surgery are generally attributed to the lower surgical trauma to the abdominal wall and potentially to the immune system.

In their paper, Imai et al. report their 20-year experience with (staged) liver resections for CRLM and surgery for tumor recurrence after two-stage hepatectomy: out of more than 1,200 resections, 139 had been performed by twostage hepatectomies for otherwise unresectable disease, and nearly all of these patients received preoperative chemotherapy (7). A third of these patients did not proceed to the second stage procedure due to tumor progression, and $75 \%$ developed tumor recurrence after potentially curative two-stage hepatectomy. The 5-year median diseasefree and overall survivals of patients who completed the two-stage procedure were $10.5 \%$ and $41.3 \%$. About half of the patients underwent re-resection for intrahepatic tumor recurrence, of whom another $50 \%$ were salvaged. Most of the patients had a single re-resection, while 12 patients had two, and three patients even had four re-resections. Repeat as well as uncomplicated surgery were independent positive prognostic factors after successful two-stage hepatectomy.

First, Imai et al. demonstrate the feasibility and safety of repeat liver resections even after extensive (staged) liver surgery in specialized centers. Second, this analysis depicts a high recurrence rate after two-stage hepatectomy, which was expected considering the risk profile of synchronous and multiple metastases. Most importantly, however, this analysis outlines, that a significant proportion of patients with primarily extensive and unresectable CRLM has a curative treatment potential by (repeat) liver surgery. Despite the rather short disease-free survival after twostage hepatectomy, this multimodality concept resulted in a 5 -year survival rate of $54 \%$ in the subgroup of patients who underwent curative re-resection for tumor recurrence. This finding emphasizes again, that the biology of CRLM differs widely: while tumor recurrence was unresectable for various reasons in some, many patients were able to undergo curative repeat surgery for limited tumor recurrence.

In summary, the Imai et al. paper is another brick in the wall of modern management of CRLM demonstrating the importance of expert liver surgery for the optimal management of patients with CRLM. As others before, this paper demonstrates that many patients even with extensive metastasis may benefit from regional treatments, although established risk scores would predict limited outcome. Ideally, patients with a rapid recurrence should receive chemotherapy and those with a favorable response should undergo aggressive surgical concepts. In the absence of sharply discriminating scoring systems, surgery should be offered to all patients with CRLM, if the disease appears resectable using all technical and medical treatment options. Although not supported by randomized trials, most patients with such extensive disease should primarily receive systemic chemotherapy as in the Imai et al. study. Upon response to this treatment, aggressive surgical concepts can be implemented. Potentially, molecular profiling will help to prospectively stratify patients to primary, staged or repeat surgery as well as chemotherapy in the future.

\section{Acknowledgments}

None.

\section{Footnote}

Conflicts of Interest: The author has no conflicts of interest to declare.

\section{References}

1. Heinrich S, Lang H. Liver metastases from colorectal cancer: technique of liver resection. J Surg Oncol 2013;107:579-84.

2. Petrowsky $H$, Gonen $M$, Jarnagin $W$, et al. Second liver resections are safe and effective treatment for recurrent hepatic metastases from colorectal cancer: a bi-institutional analysis. Ann Surg 2002;235:863-71.

3. Lang H, de Santibanes E, Schlitt HJ, et al. 10th Anniversary of ALPPS-Lessons Learned and quo Vadis. Ann Surg 2019;269:114-9.

4. Torzilli G, Adam R, Vigano L, et al. Surgery of Colorectal Liver Metastases: Pushing the Limits. Liver Cancer 2016;6:80-9.

5. Fretland AA, Dagenborg VJ, Bjornelv GMW, et al. Laparoscopic Versus Open Resection for Colorectal Liver Metastases: The OSLO-COMET Randomized Controlled Trial. Ann Surg 2018;267:199-207. 
6. Tohme S, Goswami J, Han K, et al. Minimally Invasive Resection of Colorectal Cancer Liver Metastases Leads to an Earlier Initiation of Chemotherapy Compared to Open Surgery. J Gastrointest Surg 2015;19:2199-206.

Cite this article as: Heinrich S. The current role of liver surgery in the treatment of colorectal liver metastases. HepatoBiliary Surg Nutr 2019;8(5):552-554. doi: 10.21037/ hbsn.2019.05.02
7. Imai K, Benitez CC, Allard MA, et al. Impact of Surgical Treatment for Recurrence After 2-Stage Hepatectomy for Colorectal Liver Metastases, on Patient Outcome. Ann Surg 2019;269:322-30. 